\title{
Clinical Outcomes Evaluation of Combined Valgus and Chiari Osteotomy Inconsistent with Patient Satisfaction
}

\author{
Akira Hozumi $\mathbb{D}^{D}$, Kennichi Kidera, Ko Chiba $(\mathbb{D}$, Takayuki Shida, and Makoto Osaki \\ Department of Orthopaedic Surgery, Nagasaki University Graduate School of Biomedical Sciences, Nagasaki, Japan \\ Correspondence should be addressed to Akira Hozumi; akira45512@gmail.com
}

Received 10 October 2017; Accepted 12 March 2018; Published 18 April 2018

Academic Editor: GuoXin Ni

Copyright (C) 2018 Akira Hozumi et al. This is an open access article distributed under the Creative Commons Attribution License, which permits unrestricted use, distribution, and reproduction in any medium, provided the original work is properly cited.

\begin{abstract}
The Japanese Orthopaedic Association Hip-Disease Evaluation Questionnaire, which is tailored to Japanese lifestyles, has recently been developed in Japan as a patient-reported outcome measure. In this study, combined valgus and Chiari osteotomy were evaluated using the JHEQ and JOA scores. The subjects were 42 hips of 39 patients with a mean age at surgery of 45.3 years. The mean follow-up period was 95.3 months. Radiological osteoarthritis stage, preoperative and postoperative JOA scores, JHEQ score at final follow-up, and patient dissatisfaction with hip joint status rated on a visual analog scale were evaluated. The factors that affected patient dissatisfaction were also identified using multiple regression analysis. Radiological osteoarthritis stage at final follow-up was either maintained or improved in $85.7 \%$. The mean JOA score improved from 57.2 preoperatively to 78.7 at final follow-up. The JHEQ score at final follow-up, however, was low, at 43.3 points. Patients who were comparatively satisfied accounted for $47.6 \%$. Of the JHEQ subscales, movement had the lowest scores, and this was the subscale that had the greatest effect on patient dissatisfaction. The present results suggest that the results of JOA score are inconsistent for postoperative patients' satisfaction after CVCO, and patient-based evaluation tool must also be used.
\end{abstract}

\section{Introduction}

In Japan, secondary osteoarthritis of the hip caused by developmental dysplasia of the hip (DDH) is the leading cause of osteoarthritis of the hip $[1,2]$. For the patients with primary osteoarthritis of the hip, clinical improvement is difficult to achieve even by remodeling and reconstructing the bone and hip morphology. On the other hand, improvements of the joint cartilage in DDH can be induced in many cases by the construction of biomechanical elements by osteotomy. Osteotomy has therefore been proactively used to treat comparatively young patients, even if arthritic changes are advanced. Chiari osteotomy is a surgical procedure that reduces pain by improving coverage of the acetabulum and promoting reproduction of the fibrocartilage derived from the joint capsule [2-4]. Valgus osteotomy of the hip [5-10] is a widely performed procedure to achieve joint stability, repair, and regeneration using the proliferative changes of osteoarthritis. Since 2002, we have been performing combined valgus and Chiari osteotomy (CVCO) to treat patients with advanced or terminal-stage osteoarthritis of the hip whenever possible.

Relatively good postoperative outcomes have been reported after hip osteotomy. However, they were mostly assessed by the perspective of healthcare providers. Therefore, they are not always sufficient for the more detailed evaluation of patients' physical symptoms, social hardships, and advantages as a result of surgery.

In recent years, evaluation criteria such as quality of life (QOL) and satisfaction from the patients' standpoint have been regarded as important [11-15].

The Japanese Orthopaedic Association Hip-Disease Evaluation Questionnaire (JHEQ) has recently been developed in Japan as a tool for evaluating patients' quality of life. It uses criteria specific to hip joint disease and is tailored to Japanese lifestyles. It comprises an assessment of patient dissatisfaction with hip joint status as scored on a visual analog scale (VAS) together with 20 questions, with three subscales (pain, movement, and mental status) with a possible score of 28 points each, for a total possible score of 84 points. The JHEQ is 
a patient-based evaluation tool, with each question scored by the patient on a five-point scale, and its reliability and validity have been established by Matsumoto et al. [16, 17].

In this study, the clinical outcome of CVCO was evaluated using the Japanese Orthopaedic Association hip score (JOA score) and the JHEQ, and the results were compared. Furthermore, the association of each subscale with the VAS score was also investigated. These results were then used to identify factors that affected patient dissatisfaction with this operation.

\section{Subjects and Methods}

The study subjects were 42 hips of 39 patients among the 73 patients who underwent CVCO at our hospital between January 2002 and July 2011. The JHEQ survey was selfadministered and distributed either by post or at the end of a hospital visit. The survey was sent by post only to patients who were able to undergo an examination within six months before or after it was posted.

The subjects were 4 men and 35 women, with a mean age at surgery of 45.3 years (range $22-57$ years); the mean follow-up period was 95.3 months (range 35-153 months). Preoperative stage was categorized according to the radiographic system defined in 2010 by the Investigation Group into Coxarthrosis and Acetabular Dysplasia in Japan $[18,19]$ as preosteoarthritis (2 hips), initial stage of osteoarthritis (4 hips), advanced stage of osteoarthritis (14 hips), and the terminal stage of osteoarthritis (22 hips).

The preoperative roundness index of the femoral head [20] was 59.6\%.

The items investigated were patient dissatisfaction with hip status at final follow-up (VAS), correlations between the VAS score and the JOA and JHEQ subscale scores, and correlations with other factors such as osteoarthritis stage, and the factors that had the greatest effect on the VAS score were investigated.

Statistical analysis was carried out using Spearman's rank correlation coefficient and multiple regression analysis, with $p<0.05$ regarded as significant.

This study was approved by the institutional review board (approval number: 17091101), and the requirement for informed consent was waived owing to the retrospective nature of the study.

\section{Results}

In terms of radiographic analysis at final follow-up, arthritis stage was either maintained or improved in $88 \%$ of cases, with advanced seen in only 5 hips (Figure 1).

The mean JOA score improved from 57.2 points preoperatively to 78.7 points at final follow-up (Figure 2(a)), but the total JHEQ score at final follow-up was lower, at 43.3 points (scoring rate $49.3 \%$ ) (Figure 2 (b)), lower than the JOA score. Movement subscale had the lowest scores among the various factors included in the JHEQ.

The mean VAS score at final follow-up was $43.3 \mathrm{~mm}$. Twenty patients $(47.6 \%)$, were comparatively satisfied, with a VAS score $\leq 30 \mathrm{~mm}$ (Figure 3).

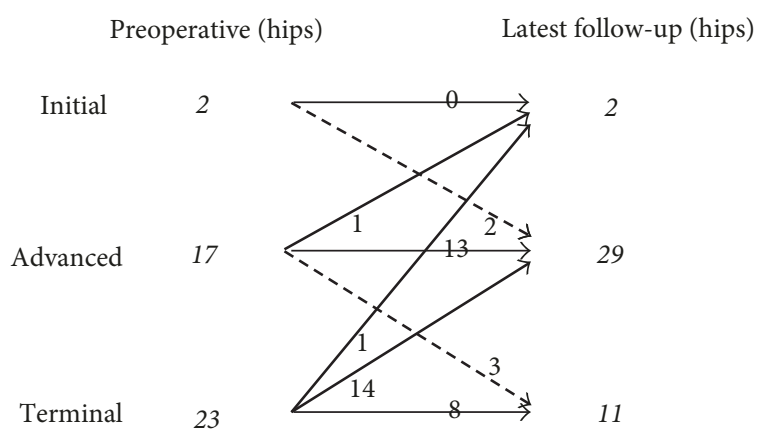

Improve: 16 hips (38\%)
Maintained: 21 hips (50\%)
Worse: 5 hips (12\%)

FIGURE 1: Changes in osteoarthritis stage. At final follow-up, osteoarthritis stage is either maintained or improved in $88.1 \%$.

TABLE 1: Correlations between JHEQ and JOA subscales.

\begin{tabular}{|c|c|c|c|c|c|}
\hline \multirow{2}{*}{ JHEQ } & \multicolumn{4}{|c|}{ JOA } & \multirow{2}{*}{ Total } \\
\hline & Pain & ROM & Gait & $\mathrm{ADL}$ & \\
\hline Pain & 0.655 & 0.509 & 0.596 & 0.564 & - \\
\hline Movement & 0.538 & 0.662 & 0.648 & 0.581 & - \\
\hline Mental & 0.626 & 0.509 & 0.655 & 0.534 & - \\
\hline Total & - & - & - & - & 0.838 \\
\hline
\end{tabular}

The value (modulus) is Spearman's rank correlation coefficient.

There was some correlation with each factor between the JHEQ and JOA scores, but they did not have the strong correlation $(\geq 0.7)$ (Table 1$)$.

In the correlation of VAS and each subscale of JHEQ and JOA, movement, mental status subscale, and total scores of JHEQ had strong correlations $(\geq 0.8)$ (Table 1$)$.

According to the correlations between the VAS score and the roundness index of the femoral head, preoperative osteoarthritis stage, osteoarthritis stage at final follow-up, age at surgery, follow-up period, and the preoperative JOA subscale score, only the osteoarthritis stage at final follow-up had a weak correlation (Table 2). Based on these correlation results, multiple regression analysis with JHEQ subscales as explanatory variables was performed to investigate their effects on the VAS score. The movement subscale had the greatest effect on VAS score (Table 3). Specifically, the mean scores for 6 of the 7 items that make up the movement subscale were $<2$ points, indicating some sort of difficulty (Table 4). For questions in the mental status subscale, the scores which influenced daily activity tended to be lower because of hip disease (data not shown).

\section{Discussion}

Recent reports have pointed out the importance of including subjective assessments from the patient's perspective in postoperative evaluation, in addition to assessment by healthcare providers, and the existence of discrepancies between these two methods of evaluation has been demonstrated. McGee 


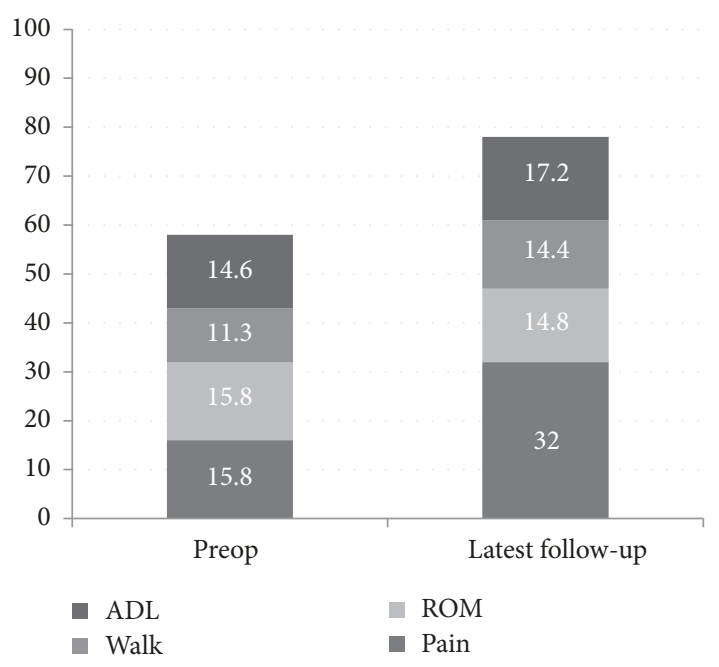

(a)

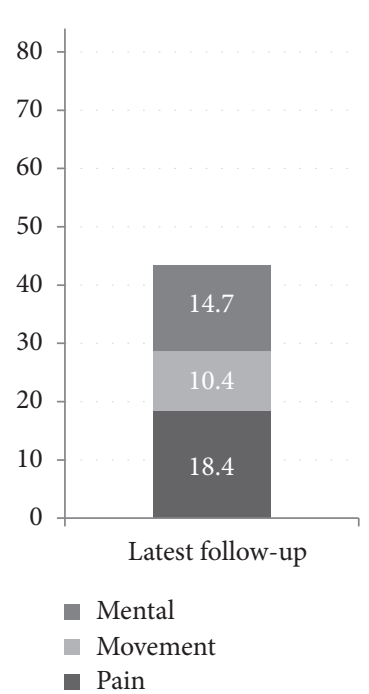

(b)

FIGURE 2: Change in JOA score and JHEQ score VAS score at final follow-up.

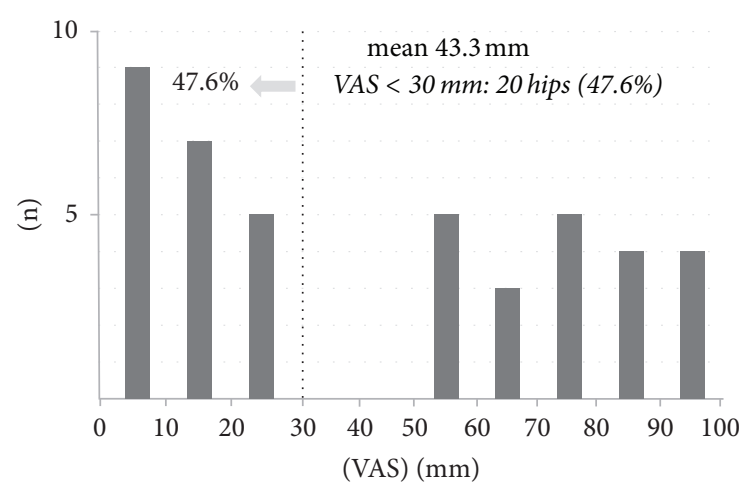

FIgURE 3: VAS score at final follow-up. The mean VAS score is $43.3 \mathrm{~mm}$, and 20 hips (47.6\%) are comparatively satisfied, with a VAS score $\leq 30 \mathrm{~mm}$.

et al. [14] found that discrepancies between objective and subjective assessments tend to emerge when patients who had undergone total hip arthroplasty had systemic complications. Liberrmann et al. [15] reported that discrepancies tended to be more common in patients with low levels of postoperative satisfaction.

In this study, assessment by the JHEQ clearly indicated a poorer outcome than when the JOA score was used, and although there was some degree of correlation between subscales, these correlations were not strong. This result was similar to those of studies reported by other authors.

In particular, the present findings suggested that the results of the JHEQ better reflect factors including the following: (1) JOA and JHEQ scores differed with respect to movement-related items that required deep hip flexion; (2) the effect on mental status of the gap between excessive expectations of surgery and the reality; and (3) the effect on the movement subscale of the negative effect on the other joints by a persistent or increased leg length discrepancy.
TABLE 2: Correlations between VAS scores and individual factors.

\begin{tabular}{lc}
\hline Factor & $\begin{array}{c}\text { Correlation } \\
\text { coefficient }(r)^{*}\end{array}$ \\
\hline Roundness index of the femoral head & 0.057 \\
Stage of osteoarthritis (preop) & 0.040 \\
Stage of osteoarthritis (latest follow-up) & 0.515 \\
Age & 0.014 \\
Leg length discrepancy (latest follow-up) & 0.349 \\
Duration of follow-up & 0.006 \\
JOA score (preoperative) & \\
Pain & 0.102 \\
ROM & 0.007 \\
Walk & 0.077 \\
ADL & 0.107 \\
\hline
\end{tabular}

${ }^{*}$ The value (modulus) is Spearman's rank correlation coefficient.

Although there have been few reports of postosteotomy patient-based assessments, Kaneuji et al. [21] looked at longterm outcomes after rotational acetabular osteotomy (RAO). They reported that the mean JHEQ score at final follow-up was 57 points, and mean patient dissatisfaction as measured by the VAS score was $22.8 \mathrm{~mm}$, which were better outcomes than in the present study. This may have been due to the different preoperative factors prevalent in CVCO compared with RAO. CVCO is often carried out on patients whose preoperative stage is comparatively advanced, and although the surgery does provide some degree of pain relief, patients still have poor hip function and low levels of postoperative activity compared with those who undergo RAO. Furthermore, it cannot provide leg length adjustment, meaning that limping may persist or even worsen over time. This may affect the other joints, which in turn affects the movement subscale. It also reflects the fact that, although this operation 
TABLE 3: Factors affecting the VAS score (multiple regression analysis).

\begin{tabular}{lcccc}
\hline & Regression coefficient & Standard error & p value & 95\% CI \\
\hline Constant term & 96.2 & 6.56 & $<0.01$ & $82.74-109.3$ \\
Pain & -0.22 & 0.60 & 0.71 & $-1.44-0.99$ \\
Movement & -2.21 & 0.74 & 0.004 & $-3.71--0.71$ \\
Mental & -1.77 & 0.57 & 0.003 & $-2.94--0.61$ \\
\hline
\end{tabular}

TABLE 4: JHEQ items (movement).

\begin{tabular}{lc}
\hline Question (movement) & Points $^{*}$ \\
\hline It is difficult for me to climb up and down stairs & 1.64 \\
It is difficult for me to get up from the floor and tatami & 1.59 \\
It is difficult for me to squat & 1.54 \\
It is difficult for me to use a Japanese-style toilet & 1.02 \\
It is difficult to get in and out of a bathtub & 2.00 \\
It is difficult to cut my toenails & 1.14 \\
It is difficult to put on my socks & 1.54 \\
\hline
\end{tabular}

${ }^{*}$ Patients' answers-strongly agree, agree, uncertain, disagree, and strongly disagree-are scored as $0,1,2,3$, and 4 points, respectively.

is time-consuming and physically invasive, it is also timesaving surgery, and factors such as the psychological anxiety and stress entailed in having to undergo a second operation. These factors may have an effect on mental status. Kaneuji et al. [21] carried out an analysis by preoperative osteoarthritis stage, and they found that those who underwent RAO for advanced osteoarthritis had significantly lower scores only on the movement subscale, a similar tendency to that observed in the present study.

Actual subjective comments received from patients included a comparatively large proportion of movementrelated complaints that affected activity levels, such as back pain from limping or difference in leg length, and increased pain in the joints of the opposite leg following surgery.

These findings suggest that the JOA score alone is insufficient for postoperative evaluation of $\mathrm{CVCO}$, and another patient-based evaluation tool such as the JHEQ must also be used. Due to recent improvements in the durability of materials and advances in surgical procedures for total hip arthroplasty, CVCO is now being used to treat younger patients, and the number of patients for whom it is indicated is tending to decrease. Further studies are required to clarify the indications for this procedure.

A limitation of this study is that the JHEQ score is difficult to use for preoperative and continuous evaluation.

\section{Conclusions}

The JOA and JHEQ were used to evaluate clinical outcomes of CVCO, and they were compared as methods of postoperative assessment. The movement and mental status subscales of the JHEQ were strongly correlated with the VAS score.

The present results suggest that an objective evaluation tool from the healthcare providers' perspective is inadequate for postoperative assessment after $\mathrm{CVCO}$, and a patientbased evaluation tool must also be used.

\section{Conflicts of Interest}

The authors declare that there are no conflicts of interest regarding the publication of this paper.

\section{Acknowledgments}

The authors would like to thank emeritus Professor, Dr. Shindo, for his help in preparing this manuscript.

\section{References}

[1] S. Jingushi, S. Ohfuji, and M. Sofue, "Multiinstitutional epidemiological study regarding osteoarthritis of the hip in Japan," Journal of Orthopaedic Science, vol. 15, no. 5, pp. 626-631, 2010.

[2] S. Nakamura, S. Ninomiya, and T. Nakamura, "Primary osteoarthritis of the hip joint in japan," Clinical Orthopaedics and Related Research, vol. 241, pp. 190-196, 1989.

[3] K. Koyama, F. Higuchi, and A. Inoue, "Modified Chiari osteotomy for arthrosis after Perthes' disease. 14 hips followed for 2-12 years," Acta Orthopaedica, vol. 69, no. 2, pp. 129-132, 1998.

[4] S. Hiranuma, F. Higuchi, A. Inoue, and M. Miyazaki, "Changes in the interposed capsule after Chiari osteotomy: an experimental study on rabbits with acetabular dysplasia," The Journal of Bone and Joint Surgery, vol. 74, no. 3, pp. 463-467, 1992.

[5] A. Inoue, "Pelvic osteotomies in the treatment of coxarthrosiswith special reference to Chiari pelvic osteotomy," Nihon Seikeigeka Gakkai Zasshi, vol. 63, no. 12, pp. 1587-1598, 1989.

[6] G. L. Maistrelli, M. Gerundini, U. Fusco, R. Bombelli, M. Bombelli, and A. Avai, "Valgus-extension osteotomy for osteoarthritis of the hip. Indications and long-term results," The Journal of Bone \& Joint Surgery (British Volume), vol. 72, no. 4, pp. 653-657, 1990.

[7] S. Jingushi, Y. Sugioka, Y. Noguchi, H. Miura, and Y. Iwamoto, "Transtrochanteric valgus osteotomy for the treatment of osteoarthritis of the hip secondary to acetabular dysplasia," The Journal of Bone and Joint Surgery, vol. 84, no. 4, pp. 535-539, 2002.

[8] S. Takasaki, K. Uchiyama, N. Takahira, and M. Itoman, "Results and prognostic factors of valgus osteotomy in middle-aged patients with advanced or terminal osteoarthritis of the hip," Journal of Orthopaedic Science, vol. 15, no. 1, pp. 20-29, 2010.

[9] M. Itoman, K. Yonemoto, M. Sekiguchi, and M. Yamamoto, "Valgus-flexion osteotomy for middle-aged patients with advanced osteoarthritis of the hip: a clinical and radiological evaluation," Nihon Seikeigeka Gakkai Zasshi, vol. 66, no. 4, pp. 195-204, 1992.

[10] M. Itoman, M. Yamamoto, K. Yonemoto, M. Sekiguchi, and H. Kai, "Histological examination of surface repair tissue after successful osteotomy for osteoarthritis of the hip joint," International Orthopaedics, vol. 16, no. 2, pp. 118-121, 1992. 
[11] J. B. T. Lim, A. C. C. Chou, W. Yeo et al., "Comparison of patient quality of life scores and satisfaction after common orthopedic surgical interventions," European Journal of Orthopaedic Surgery and Traumatology, vol. 25, no. 6, pp. 1007-1012, 2015.

[12] J. Klit, "Results of total joint arthroplasty and joint preserving surgery in younger patients evaluated by alternative outcome measures," Danish Medical Journal, vol. 61, no. 4, p. B4836, 2014.

[13] J. Klit, C. Hartig-Andreasen, S. Jacobsen, K. Søballe, and A. Troelsen, "Periacetabular osteotomy: sporting, social and sexual activity 9-12 years post surgery," Hip International, vol. 24, no. 1, pp. 27-31, 2014.

[14] M. A. McGee, D. W. Howie, P. Ryan, J. R. Moss, and O. T. Holubowycz, "Comparison of patient and doctor responses to a total hip arthroplasty clinical evaluation questionnaire," The Journal of Bone \& Joint Surgery, vol. 84, no. 10, pp. 1745-1752, 2002.

[15] J. Liberrmann et al., "Difference between patients and physicians evaluation of outcome after total hip arthroplasty," The Journal of Bone and Joint Surgery American, vol. 78, pp. 835-838, 1996.

[16] T. Matsumoto, A. Kaneuji, Y. Hiejima et al., "The Subcommittee on Hip Disease Evaluation of the Clinical Outcome Committee of the Japanese Orthopaedic Association. Japanese Orthopaedic Association Hip Disease Evaluation Questionnaire (JHEQ): a patient-based evaluation tool for hip-joint disease," Journal of Orthopaedic Science, vol. 17, no. 1, pp. 25-38, 2012.

[17] T. Seki, Y. Hasegawa, K. Ikeuchi, N. Ishiguro, and Y. Hiejima, "Reliability and validity of the Japanese Orthopaedic Association hip disease evaluation questionnaire (JHEQ) for patients with hip disease," Journal of Orthopaedic Science, vol. 18, no. 5, pp. 782-787, 2013.

[18] R. Ogawa and S. Imura, "Evaluation chart of hip joint functions," Nippon Seikeigeka Gakkai Zasshi, vol. 69, p. 860, 1995 (Japanese).

[19] Y. Takatori, K. Ito, M. Sofue et al., "Analysis of interobserver reliability for radiographic staging of coxarthrosis and indexes of acetabular dysplasia: a preliminary study," Journal of Orthopaedic Science, vol. 15, no. 1, pp. 14-19, 2010.

[20] T. Savaridas, R. J. Wallace, D. M. Salter, and A. H. R. W. Simpson, "Do bisphosphonates inhibit direct fracture healing? A laboratory investigation using an animal model," The Bone \& Joint Journal, vol. 95, no. 9, pp. 1263-1268, 2013.

[21] A. Kaneuji, T. Sugimori, T. Ichiseki, K. Fukui, E. Takahashi, and T. Matsumoto, "Rotational acetabular osteotomy for osteoarthritis with acetabular dysplasia: conversion rate to total hip arthroplasty within twenty years and osteoarthritis progression after a minimum of twenty years," The Journal of Bone and Joint Surgery, vol. 97, no. 9, pp. 726-732, 2015. 


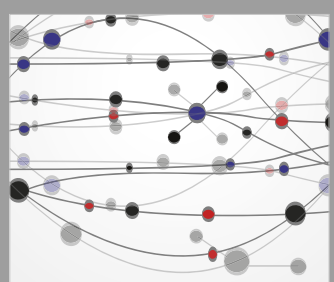

The Scientific World Journal
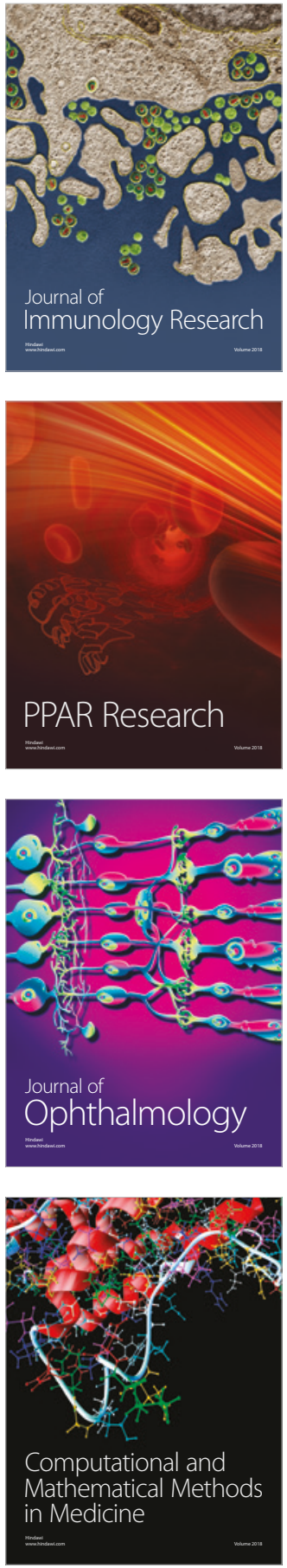

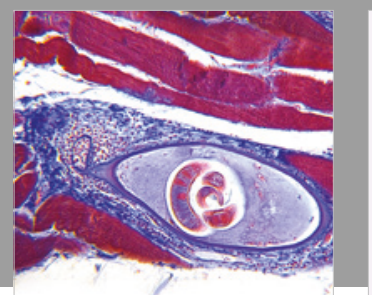

Gastroenterology Research and Practice

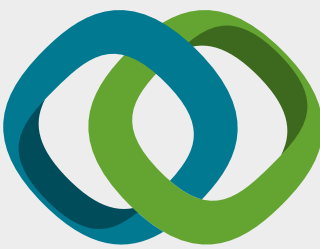

\section{Hindawi}

Submit your manuscripts at

www.hindawi.com
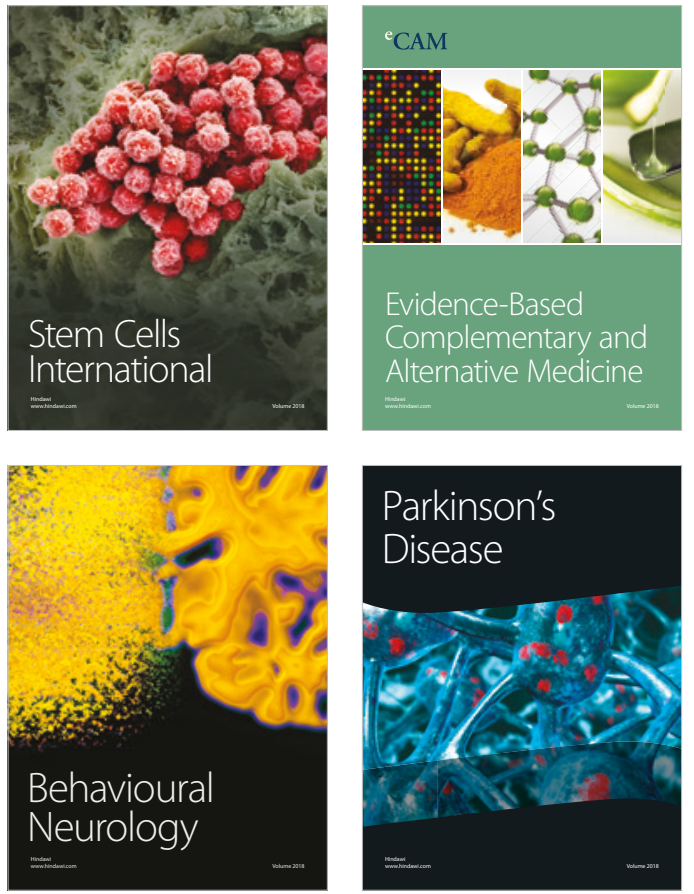

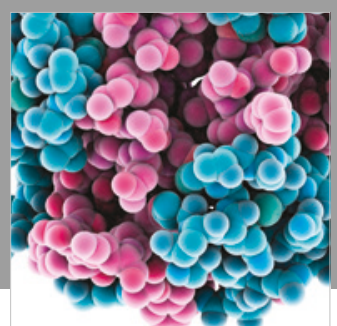

ournal of

Diabetes Research

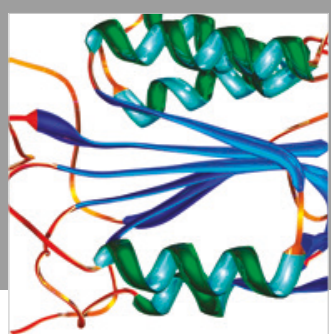

Disease Markers
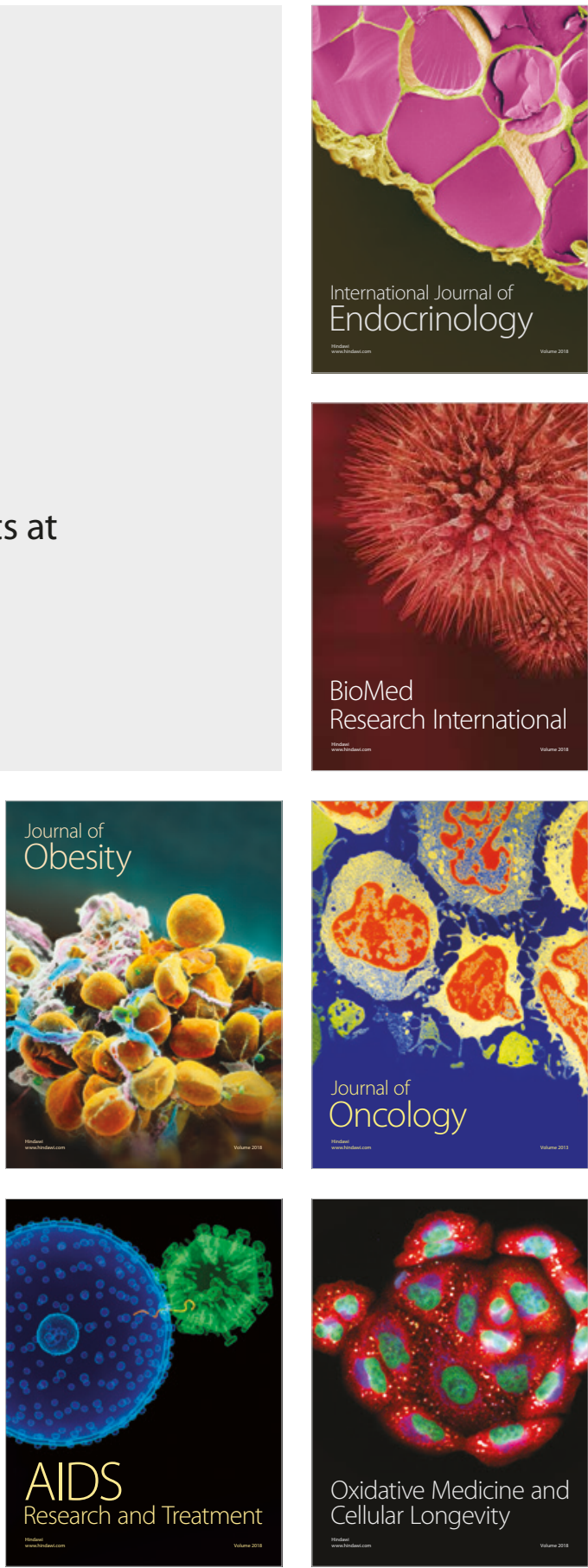